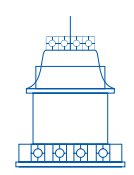

RESEÑ A

\title{
Wendland, J., Merwin, C. y Hadjioannou, C. (Eds.). (2018). Heidegger on technology. New York: Routledge.
}

\author{
Omar Camilo Moreno Caro \\ Departamento de Filosofía, Universidad Nacional de Colombia, Bogotá, Colombia \\ E-mail: camil69@hotmail.es | ORCID: 0000-0002-6931-8688 \\ https://doi.org/10.17533/udea.ef.n61a13
}

En muchos sentidos diferentes, la crisis mundial actual (los enfrentamientos en medio oriente, la inestabilidad política y social de Latinoamérica, las múltiples migraciones, el resurgimiento de los fascismos en los países europeos, el cambio climático, entre otros) no es propiamente un síntoma del fracaso del proyecto ilustrado occidental y de sus instituciones. Desde una perspectiva heideggeriana, se trata de un problema ontológico, de una crisis en el acercamiento técnico al mundo que se hace manifiesta gracias a las fuertes tensiones internas del ideal del progreso. Sin embargo, no podemos hablar propiamente de una crisis en la tecnología. Basta ver los impresionantes desarrollos de los últimos 20 años para comprobar que estamos en la época más radicalmente técnica que ha existido jamás. Desde los computadores personales hasta las modificaciones en el genoma humano son muestras y garantías del efectivo desarrollo y de las grandes promesas del desarrollo científico e industrial. Entonces cobra sentido la pregunta ¿qué significa preguntar por la esencia de la técnica, es decir, del acercamiento técnico al mundo?, ¿cuál es el problema con la tecnología?

En primer lugar, la esencia de técnica no se refiere al rasgo común de la diversidad de dispositivos tecnológicos o de su conjunto, más bien, esta refiere a cierto tipo de relación en la que se encuentra el ser humano con el mundo, una determinada forma de apertura o una configuración de la verdad $(a \lambda \dot{\eta} \theta \varepsilon l \alpha) .{ }^{1}$ Heidegger describe

1 La noción de verdad que sirve como base de la meditación heideggeriana sobre la técnica tiene un complejo entramado de relaciones 
esta relación como Gestell, una palabra con la que busca referirse a la articulación de los múltiples comportamientos del ser humano (disponer, almacenar, presupuestar, consumir, entre otros) cuyo destino es hacer de la naturaleza un mero medio disponible para nuestro uso y, consecuentemente, la disposición de nosotros mismos para ser utilizados como recursos. Para Heidegger, esta "organización" no es una actitud o una decisión propia de los gobernantes, los científicos o los ingenieros, más bien refiere a determinada forma de estar abierto al mundo que se desarrolla en todos los ámbitos de la vida y en la que se consuma la metafísica occidental, a saber, la voluntad de imposición sobre el mundo, la naturaleza y los seres humanos.

De este modo, en el presente la crisis no es científica ni el problema es solo una futura escasez de recursos básicos, se trata de una crisis en la forma en que nos relacionamos con el mundo: independientemente de la situación, cada vez la Gestell determina más profundamente nuestro encuentro con los entes y cada vez es más violento su despliegue (basta ver los incendios del Amazonas para entender lo que está en juego). Además, en el proceso de hacer a la naturaleza un objeto (Gegenstand) o una mercancía en stock (Bestand), cada vez más se oculta la posibilidad de establecer una relación libre con ella. Con esto, inevitablemente nos estamos perdiendo a nosotros mismos, pues poco a poco somos reducidos a nuestra mera disponibilidad de tiempo y de recursos. Esta forma de relación no solo se replica en el mercado o en las relaciones humanas (donde es más evidente), tiene profundas consecuencias en la política (por ejemplo, la burocracia y la tecnocracia) o de manera más potente en la economía actual.

Sin embargo, "Donde está el peligro, crece también lo que salva" reza el verso de Patmos, el poema de Hölderlin que, para Heidegger, ayuda a entender y afrontar la época más radicalmente peligrosa de todas y, por lo tanto, en la que es posible la mayor salvación. Ante este impulso irrefrenable de control y cálculo total de la naturaleza y de la existencia, el filósofo alemán propone la Gelassenheit o aquello que ha sido traducido al español como serenidad. En otras palabras, frente al despliegue enloquecido de la pulsión por disponerlo todo como consumible o mercancía (la naturaleza, el amor, el odio, la política, el bien, la violencia, las conciencias, los nacimientos y la muerte), Heidegger afirma sorpresivamente que la actitud que representa una respuesta adecuada a esta forma de verdad ( $a \lambda \dot{\eta} \theta \varepsilon \imath \alpha)$ es la serenidad, dejar ser lo que es.

¿Significa esto una total pasividad frente al impulso violento y objetivizante de la Gestell?, ¿se trata de cruzar los brazos y esperar el fin de la técnica (o el fin del mundo)

y consecuencias que es imposible desarrollar aquí, sin embargo, vale la pena hacer un par de precisiones. El concepto de verdad $(a \lambda \dot{\eta} \theta \varepsilon l \alpha)$ que se utiliza para caracterizar la esencia de la técnica no corresponde al concepto de verdad como correspondencia ni a los múltiples sentidos de verdad que se utilizan en la labor científica. Más bien, verdad refiere a una dinámica concreta que hace posible el develamiento de los entes y que se configura en cada época de una determinada manera. En ese sentido, que la técnica sea una forma de verdad significa que se trata de una manera especíica en que el mundo se hace comprensible para el ser humano o una forma muy concreta en la que se desarrolla la relación con el mundo que, en el presente, se encuentra dirigida por la Gestell o imposición. Para un estudio detallado de la doctrina de la esencia y la verdad en la meditación sobre la técnica, recomiendo acudir al estudio de D. Dahlstrom contenido en este compendio: "Im-position: Heidegger's Analisis of the Essence of Modern Technology". 
retirados en la montaña o en la selva negra? De ninguna manera. En un tiempo tan revotado por revoluciones tecnológicas y sediento de poder y de consumo, quizá la verdadera resistencia sea dejar ser al imperio de la Gestell en vez de asumir una actitud de control sobre la tecnología que, con seguridad, solo radicalizaría su impacto sobre la vida humana y la naturaleza. Es probable que los abundantes intentos de poner límites a los desarrollos tecnológicos y a la investigación no sean más que otra manifestación de la lucha por el poderío tecnológico y, aunque parezcan un giro de tuerca, solo confirman y acentúan la ansiedad general por el control. Pero, en el fondo lo que verdaderamente significa todo esto es un enigma: ¿a qué se refiere propiamente la Gelassenheit?, ¿está en juego aquí una forma radicalmente nueva de comprender la acción y la libertad?, ¿es posible en nuestro presente una meditación lo suficientemente radical sobre la técnica?, ¿cuáles son las formas contemporáneas de la Gelassenheit?

Heidegger on technology es un compendio de estudios que asumen el reto de enfrentar estas preguntas desde diversos horizontes. Esta serie de trabajos busca ofrecer un panorama completo de las consideraciones teóricas, metodológicas, prácticas y políticas que se desprenden de la meditación sobre la esencia de la técnica. Esto es profundamente pertinente luego de la publicación de algunos textos originales de Heidegger que ayudan a dar respuesta a algunos interrogantes importantes que habían dejado La pregunta por la esencia de la técnica y Discourse on thinking. A continuación, me detendré en algunas preguntas claves que ayudan a articular el libro como una continuación de un diálogo que comienza justamente en la pregunta por la técnica. Para esto me concentraré en algunos artículos que sirven como nodo para hilar las otras contribuciones que hacen parte de este compendio alrededor de algunas preguntas cruciales.

\section{¿Qué significa pensar en la época técnica?}

El capítulo "The Task of Thinking in a Technological Age" de M. Wrathall ofrece las bases para entender este problema, en especial porque se centra en hacer un detallado examen de la tesis básica a partir de la que se desarrolla la indagación heideggeriana. Cuando se reconoce que la técnica es una forma (dentro de otras) de relacionarse con el mundo que se puede describir como Gestell, la primera pregunta que surge es si todavía se pueden identificar otras formas de develamiento $(a \lambda \dot{\eta} \theta \varepsilon \imath \alpha)$ en el presente, por ejemplo, en ciertos grupos indígenas o en determinadas comunidades. Efectivamente Heidegger señala que los modos de develamiento son universales (alcanzan todos los entes en su singularidad y a todos los modos de acercamiento) y totales (no dependen de nuestra voluntad particular, pues la verdad no es algo propiamente humano). Sin embargo, ¿esto es una descripción adecuada de la realidad?, ¿acaso no es exagerado y poco plausible pensar que existe un único modo de relacionarse con el mundo en nuestra época? 
Sin más precisiones, esta tesis parece sugerir que el paso del tiempo implica épocas del mundo que se van desarrollando sin mayor participación humana y que condicionan toda acción posible. De este modo, la primera crítica que se suele hacer a este camino del pensar es que se trata de un planteamiento radicalmente fatalista y que su respuesta, la Gelassenheit, solo invita a la inercia política y social. Otra crítica importante es que, en la medida en que se trata de un acercamiento universal a los entes, el punto de partida de Heidegger es ontológicamente conservador, pues no esclarece cómo es posible la multiplicidad de formas de relacionarnos con el mundo o su transmutación, lo que lo acerca peligrosamente con la astrología y el misticismo.

Wrathall señala que para responder a estas críticas es crucial esclarecer el despliegue óntico de estas formas de desocultamiento, es decir, es necesario entender la dinámica concreta de la verdad y sus manifestaciones efectivas. Sin embargo, esto no significa un examen sociológico ni histórico de los modos en que ha sido posible la configuración de una determinada verdad, más bien, es necesario reconocer adecuadamente el alcance de la universalidad y totalidad de las formas de develamiento. En primer lugar, Heidegger encuentra que es posible que dos formas diferentes de abrirse al mundo convivan sincrónicamente. Esto significa que la universalidad de la que hablamos no se refiere a una postura sobre cómo son las cosas, sino a la forma en la que se articula su venir a la presencia o su inteligibilidad. ${ }^{2}$ La Gestell no es un tiempo del mundo, sino una situación concreta del despliegue de una determinada tradición que lentamente busca imponerse sobre todas las demás. Para Wrathall, lo que se encuentra en la base de este impulso es común con todos los otros modos históricos de verdad, a saber, un cierto tipo de "llamado" (claim) o "demanda" (demand) que coordina el venir a la presencia de todos los entes a partir de un destino (Geschick). En el caso de la época técnica este destino es la disponibilidad del ente como mercancía o recurso (Bestand). Entonces, la universalidad de la que hablamos señala que, bajo el yugo de un determinado modo de develamiento, todas las entidades deben poder ser inteligibles a la luz de un principio articulador de la realidad, incluso si no ocurre efectivamente. ${ }^{3}$ Esto no excluye que existan grupos humanos que no hayan sido tocados por esta tradición cultural, no obstante, no hay que olvidar que el destino de poderío y control de la Gestell apunta, en todo caso, a ser un modo totalitario de relación con el mundo de alcance planetario.

2 Es importante reconocer la resonancia fenomenológica que tiene esta tesis y esta indagación. En el fondo, el camino del pensar que se le abre a Heidegger con esta indagación supone un trasfondo fenomenológico muy importante, pues la meditación sobre la esencia no es otra cosa que una respuesta a la pregunta por las condiciones de inteligibilidad del mundo. Estos horizontes son desarrollados a profundidad por Christos Hadjioannou en el capítulo "Heidegger's Cririque of Techno-science as a Critique of Husserl's Reductive Method" y por Steven Crowell en "The Challenge of Heidegger's Approach to Technology: A Phenomenológical Reading".

3 Este punto es muy importante para entender la resonancia subjetiva de la verdad, es decir, la forma en que una determinada época histórica da lugar a los comportamientos humanos. Ciertamente podemos seguir comportándonos como si el mundo no fuera develado de forma técnica y asumir una especie de ingenuidad tecnológica. Incluso podemos huir de las ciudades. 
Luego de esta explicación, se esclarece un posible camino para entender la crítica fatalista. Es claro que el despliegue de una determinada forma de acercarse al mundo se articula a partir de una serie de prácticas que hacen posible una determinada forma de venir a la presencia. Podríamos hablar en este caso de un determinado tipo de administración, una forma concreta de producción y una manera específica de entender las relaciones humanas. Todas estas se encuentran previamente articuladas a la luz del destino de la Gestell. Según Wrathall, esto nos revela que una determinada forma de relacionarse con el mundo se sostiene sobre unas prácticas concretas que hacen posible que un determinado modo de acercarse al mundo sea universal y total. Una forma de confrontar la Gestell es justamente desarrollar prácticas que preparen el camino a una nueva forma de estar abiertos al mundo, este es el sentido de pensar (Gedenken) y de la Gelassenheit: abrir caminos que ayuden a preparar una nueva venida a la presencia en la que el mundo y la humanidad no se encuentren en peligro constante de ser reducidos a una mercancía en un almacén o a un proletariado de reserva.

\section{¿Cómo se consolida en la actualidad la Gestell? ¿Cómo es una educación que responde al llamado de la Gelassenheit?}

Ian Thompson se enfrenta a esta polémica cuestión en el capítulo "Technology, Ontotheology, Education". Una respuesta adecuada a la época técnica implica reconocer, como señala Reinner Schürmann (1987), una nueva forma de relación entre el pensamiento y la acción. Esto significa que, en la búsqueda de nuevos caminos que Ileven a una época post-tecnológica, es crucial evitar el impulso técnico por controlar nuestra relación con el mundo, pues la que parece la respuesta obvia y necesaria (un "cambio de actitud") no hace más que agudizar el verdadero problema: el imperio del control y de la planificación. De este modo, la Gelassenheit debe ser una actitud necesariamente preparatoria que busca, más allá de lograr un determinado resultado (la superación de la metafísica, la solución de los males del mundo, etc.), sentar las

Sin embargo, el problema es que el verdadero poder que ejerce sobre nosotros una época radica en que articula nuestros posibles comportamientos con respecto al ente y a nosotros mismos. Justamente podemos pretender "cambiar de actitud" porque lo que se encuentra en la base es una actitud técnica con respecto a nuestra situación y a nuestras propias "decisiones". Sobre este asunto el trabajo de Wrathall es revelador, pues no interpreta el resultado óntico de esta determinada forma de venir a la presencia exclusivamente como reservas o recursos en stock, sino como opciones (options). De este modo, la pretensión de "cambio de actitud" frente a la técnica solo es posible en el marco de una época donde incluso la verdad (como forma concreta de develamiento) aparece como un asunto que puede estar bajo nuestro control y que depende de nuestra decisión subjetiva. En este sentido, huir de la tecnología no es muy diferente de dirigir un centro de investigaciones en una multinacional de producción agrícola. Un estudio incluido en este volumen que ayuda a lograr una mejor perspectiva de esta manifestación óntica, pero desde la perspectiva de la máquina, lo ofrece A. Mitchell en "The Question Concerning the Machine: Heidegger's Technology Notebooks in the 1940-1950s". 
bases de una práctica precursora de otro modo de venir a la presencia del ente. ${ }^{4}$ En este contexto, la pregunta por una educación precursora de una nueva venida a la presencia es muy pertinente, pues esta es el ámbito en el que pueden surgir aquellas prácticas que abrirán la posibilidad de una relación postecnológica con el mundo. Entonces ¿cómo es una educación que responda adecuadamente los retos de una época radicalmente técnica?

Para enfrentar esta cuestión, Thomson recuerda la importancia de entender a fondo la crítica que hace Heidegger al nihilismo tecnológico, esto significa, entre otras cosas, reconocer su indudable dependencia con la tesis de la ontoteología occidental. Solo si reconocemos la relación entre estos dos impulsos del pensamiento heideggeriano será posible entender las exigencias propias de una educación de este tipo. La ontoteología es una forma de describir el doble núcleo que ha guiado el despliegue de la filosofía en occidente en su intento de dar una explicación definitiva de la realidad: por una parte, la reflexión metafísica (ontológica) que busca sentar un fundamento adecuado a la experiencia humana (todas las grandes filosofías participan de este núcleo, por ejemplo, Platón, Descartes, Kant, entre otros); por otra, un núcleo teológico que explica cómo estas teorías logran desplegarse en la realidad, es decir, una respectiva cosmología que avala dichas ontologías (por ejemplo, el Dios cristiano, la sustancia hegeliana o el eterno retorno nietzscheano). Las diferentes formas de articular ambos núcleos, su vigor y su decadencia han marcado el despliegue histórico de las épocas hasta el presente técnico. Este último modo de venir a la presencia es el que más ha tenido oportunidad de brindar un fundamento último a la experiencia. Sin embargo, esto significa también que se trata del modo más violento y peligroso, pues ante él estamos en riesgo de perder la posibilidad de tomar otros caminos para traer el mundo ante nosotros.

De este modo, la ontoteología ha sido el sendero de una ontología totalitaria del ente, es decir, de una respuesta definitiva a la existencia. La forma en que se ha consumado este camino es la Gestell, el peligro más grande. Se trata del mayor peligro porque lo que está en juego es nuestra esencia, la existencia. Una respuesta definitiva al ser privaría al hombre de su libertad de abrir nuevos caminos. ${ }^{5}$ Entonces, la Gelassenheit debe responder al reto de cultivar otros modos de existencia que corroboren que la apertura técnica no puede ser tan total y tan decisiva como ella pretende. Sin embargo, el reto es mayor de lo que parece, pues no se trata de diseñar nuevos estilos de vida

4 Vale la pena reconocer la complejidad que encierra este concepto desde las múltiples dificultades que ofrece la meditación sobre la técnica, solo así es posible lograr entender en qué consiste la propuesta de Heidegger. Bret Davis en "Heidegger Releasement From the Technological Will" nos ofrece un estudio detallado de la conexión que existe entre las exigencias que implica el diagnóstico heideggeriano y la respuesta que encuentra el pensador en la Gelassenheit. Aquí también valdría la pena consultar el capítulo "Heidegger's New Beginning: History, Technology, and National Socialism" de Aaron Wendland y "Poetry and the Gods: From Gestell to Gelassenheit" de Susanne Claxton.

5 El concepto de libertad que está en juego en estas meditaciones es muy complejo e importante para entender el sentido general de lograr "una relación libre" con la esencia de la técnica, a saber, la Gelassenheit. Este problema es desarrollado de forma adecuada por Julian Young en Heidegger, Habermas, Freedom, and Technology. 
o de dar lugar a nuevas comunidades o formas de integración social. ${ }^{6}$ El problema es mucho más profundo. Lo que está en juego no es apenas un orden cultural, sino aquello que se encuentra en la base de toda ciencia, cultura, ética y política. ${ }^{7}$ Se trata de un movimiento fundamental por cuanto configura radicalmente nuestros diversos acercamientos al mundo. Así, una nueva pedagogía no puede ser pensada como una mera técnica de enseñanza y aprendizaje de métodos de producción o de investigación, es necesario reconocer que, en principio, esta nueva educación debe partir por preguntar qué significa aprender y cómo es posible aprender a pensar.

Si el peligro del nihilismo tecnológico es producir una apertura que determine completamente nuestras posibilidades con respecto al ente, la salvación se encuentra en el origen de dicho peligro, a saber, en el hecho de que solo es posible establecer una determinada apertura al ente sobre la base de la movilidad histórica del ser humano y su relación con el mundo. La condición de posibilidad de una determinada época de la verdad, v.g. la Gestell, es justamente el ámbito previo desde el que se establece cada época. En este contexto, el camino que abre la meditación sobre la técnica implica aprender a revelar este ámbito previo y a performarlo. Dicho aprendizaje es diferente a lo que se reconoce institucionalmente, pues propiamente no es la trasmisión de un conocimiento sobre algo, más bien se trata del cultivo de una disposición que permite ver otras posibilidades allí donde la Gestell impone una respuesta definitiva, implica generar la posibilidad de nuevas formas de ver que abren espacio a nuevos caminos del pensar. El estudio de Tompson es muy sugerente, pues al revelar los sustentos teóricos que sostienen la crítica al nihilismo esclarece los retos que debe enfrentar el cultivo de la Gelassenheit.

\section{¿Qué movimientos contemporáneos representan una forma de la Gelassenheit heideggeriana?}

Luego de atender a las anteriores preguntas, vale la pena considerar la distancia histórica del pensamiento heideggeriano y atender a aquellas manifestaciones que se encuentran en consonancia con su forma de responder a la interpelación técnica. Esto ayuda, no solo para encontrar posibles coincidencias y movilizaciones de nuevas formas de relacionarse con el mundo, sino primariamente a esclarecer el sentido de la problemática Gelassenheit. No hay que perder de vista que la "serenidad" a la que alude Heidegger no es un concepto transparente. Es necesario que esta propuesta se

6 Este peligro lo estudia de forma muy interesante, y en directa relación con la sociedad de la auditoría, Denis McManus en el capítulo "Machenschaft and the Audit Society: The Philosophy and Politics of 'the Accessibility of Everything to Everyone'”.

7 En este compendio hay tres estudios que se encargan de mostrar cómo se puede dar cuenta del despliegue de la Gestell en diferentes ámbitos de la experiencia como, por ejemplo, la ciencia: "Heidegger vs. Kuhn: Does Science Think?" de Aaron Wendland, "Quantum Theory as Technology” de Taylor Carman y “Naturalising Gestell?” de Rafael Winkler. 
manifieste en toda su problematicidad si queremos entender, no solo su entramado conceptual, sino los verdaderos alcances del diagnóstico y, por lo tanto, su verdadero impacto en la vida humana. Un diálogo con las posibles manifestaciones de esta forma de acercarse al mundo ayudará, entonces, a comprender más adecuadamente los problemas que esta implica.

En este sentido, hay dos estudios que vale la pena revisar porque contienen acercamientos a diferentes propuestas que de una u otra forma encarnan algunos aspectos de la Gelassenheit. El primero es de Michael Zimmerman y se titula "How Pertinent is Heidegger's Thinking for Deep Ecology?" En él se comienza por mostrar cómo las crisis ambientales y la explotación sistemática de la naturaleza no es propiamente un problema ideológico, como algunas posturas ecomarxistas sostienen, sino que se trata de una determinada comprensión del mundo y de los entes. Luego, desarrolla un estudio que busca mostrar en qué sentido la Gelassenheit ofrece una perspectiva que abre las posibilidades a nuevas formas de relacionarse con la naturaleza. Finalmente, propone varios puntos de encuentro con las propuestas de la ecología profunda, en especial aquellos que tienen que ver con la cuaternidad, ${ }^{8}$ ya que descentraliza al ser humano como agente de la verdad y lo enmarca en un complejo entramado de relaciones que lo hacen posible. En otras palabras, la Gelassenheit implica reconocer que el ser humano, pese a su primacía óntico-ontológica, no es el eje fundamental de la realidad, sino que se encuentra en un rico y complejo entramado de relaciones de dependencia con su contexto histórico-social, con su lenguaje y, especialmente, con su medio ambiente.

Otro aporte de gran relevancia lo ofrece Patricia Glazebrook en el capítulo "Lettin Beings Be: An Ecofeminist Reading of Gestell, Gelassenheit and Sustainability". Este estudio se posa sobre la idea de que uno de los momentos decisivos de la Gelassenheit tiene que ver específicamente con la relación que establece el hombre con la naturaleza. Esta perspectiva es muy rica y productiva, pues permite reinterpretar la articulación de la Gestell desde la perspectiva de los recursos energéticos, lo que significa que nuestra relación con la naturaleza en la época técnica se articula a partir de nuestra dependencia energética con respecto a ella. La sostenibilidad es un esfuerzo que busca matizar esta dependencia de modo que el ser humano no consuma más energía de la que necesita y, por lo tanto, no tenga que forzar a la naturaleza a ser su reserva inagotable de energía. De este modo, la sostenibilidad busca fomentar prácticas que permitan al ser humano generar otra forma de relacionarse con la naturaleza, lo que significa, siguiendo a Heidegger, abrir el ámbito desde el que el ser humano se relaciona con el mundo.

8 Este es un tema muy poco trabajado en lengua hispana y que tiene fuertes implicaciones en la forma en que Heidegger entiende la apertura al ente. Para entender adecuadamente a qué se refiere Zimmerman basta con saber que Heidegger señala que la apertura al mundo no es un fenómeno que se articule subjetivamente o meramente en un terreno trascendental. "Para este filósofo, además de los mortales, hay tres dinámicas diferentes que se articulan de modo que sea posible la apertura al mundo, estas son: la tierra, el cielo y los celestes" Cfr. Heidegger (2014) y Másmela (2007). 
En conclusión, este compendio muestra cómo la pregunta heideggeriana sobre la esencia de la técnica lleva las discusiones sobre la tecnología a un nuevo nivel. Esto es crucial porque desplaza el foco de los problemas actuales, al mismo tiempo que desmitifica la supuesta actitud tecnófoba de Heidegger. Queda claro que todavía hay muchas posibilidades teóricas y prácticas que esta corriente de pensamiento puede ayudar a cultivar, especialmente en la época de las inteligencias artificiales, las modificaciones al genoma humano y los viajes espaciales. Además, problematiza aquella tendencia actual que sostiene que una respuesta adecuada al descontrolado desarrollo tecnológico debe ser un giro ético en la planeación. Frente a esto sostiene que solo una transformación práctica ayudará a sentar las bases de un futuro donde la utilidad y el control no dirijan nuestra existencia a costa de la naturaleza, nuestra libertad y la barbarie de un capitalismo tecnológico cada vez más macabro y funcional.

\section{Referencias}

Heidegger, M. (2014). Construir, habitar, pensar. Cali: Lugar a dudas.

Másmela, C. (2007). Ensamblaje del mundo como cuaternidad. Diálogos, 90, 211-223.

Schürmann, R. (1987). Heidegger o Being and Acting: From principles to Anarchy. Indiana: Indiana University Press. 